VOL. 9 (1973), 259-265.

\title{
Connected expansions of topologies
}

\section{J.A. Guthrie, D.F. Reynolds, and H.E. Stone}

\begin{abstract}
This note presents a characterization of connected simple expansions of topologies in terms of conditions on subspaces, and gives corollaries improving and unifying known sufficient conditions. These results are applied to obtain information about maximally connected spaces, and it is shown that maximal connectedness is inherited by connected subspaces.
\end{abstract}

If $(X, \tau)$ is a topological space and $A$ is a family of subsets of $X$, we denote by $\tau(A)$ the topology for $X$ having $\tau \cup A$ as $i t s$ subbase. This is the expansion of $\tau$ by $A$, and is the smallest topology for $X$ containing both $\tau$ and $A$. If $A=\{A\}$, we denote by $\tau(A)$ the simple expansion $\tau(\{A\})$ of $\tau$ by $A$. We consider here the related problems of determining when an expansion of a topology is connected and when a connected topology is maximally connected, that is, has no connected proper expansion. We will make frequent use of the fact that members of $\tau(A)$ have the form $V \cup(W n A)$ for open sets $V$ and $W$ in $\tau$. Finally, if $S$ is a subset of $X$, we denote by $\tau \mid S$ the relative topology induced by $\tau$ on the subspace $S$, and by $\mathrm{Cl}(S)$ the closure of $S$ in $(X, \tau)$.

\section{Expanding connected topologies}

The following theorem characterizes connected simple expansions in terms of subspace topologies.

THEOREM 1. Let $(X, \tau)$ be connected and let $A \subseteq X$. Then $(X, \tau(A))$ is connected if and only if there exists no nontrivial $C \subseteq X$ such that alz of the following hold:

Received 3 May 1973. 
(a) $C \cap A$ belongs to $\tau \mid A$;

(b) $(X-C) \cap A$ belongs to $\tau \mid A$;

(c) $C \cap(X-A)$ belongs to $\tau \mid(X-A) \cup(X-C)$;

(a) $(X-C) \cap(X-A)$ belongs to $\tau \mid(X-A) \cup C$.

Proof. If $\tau(A)$ disconnects $X$, let $C$ be a nontrivial open and closed set. Then there are $V, V^{\prime}, W, W^{\prime}$ in $\tau$ with $C=V \cup(W n A)$, $X-C=V^{\prime} \cup\left(W^{\prime} \cap A\right)$. Clearly $C \cap A=(V \cup W) \cap A$ is in $\tau \mid A$ and $(X-C) \cap A=\left(V^{\prime} \cup W^{\prime}\right) \cap A$ is in $\tau \mid A$, so that $(a)$ and $(b)$ hold. Now $C \cap(X-A)=C \cap[(X-A) \cup(X-C)]=V \therefore[(X-A) \cup(X-C)]$ since $W \cap A \subseteq C$; hence (c) holds. Finally

$$
(X-C) \cap(X-A)=(X-C) \cap[(X-A) \cup C]=V^{\prime} \cap[(X-A) \cup C]
$$

since $\left(W^{\prime} \cap A\right) \cap C=\emptyset$. Thus (d) holds. This proves the sufficiency of the conditions.

Now suppose $C$ is a nontrivial set satisfying the conditions. By (a), $C \cap A=W \cap A$ for some $W$ in $\tau$, and by (c), $C \cap(X-A)=V \cap[(X-A) \cup(X-C)]$ for some $V$ in $\tau$. Thus $V \cup(W \cap A)=C$ is in $\tau(A)$. By $(b),(X-C) \cap A=W^{\prime} \cap A$ for some $W^{\prime}$ in $\tau$ and by (d), $(X-C) \cap(X-A)=V^{\prime} \cap[(X-A) \cup C]$ for some $V^{\prime}$ in $\tau$. Then $V^{\prime} \cup\left(W^{\prime} \cap A\right)=X-C$ is in $\tau(A)$, so that $\tau(A)$ disconnects $X$. This proves the necessity.

We present some corollaries which include and improve known sufficient conditions. Corollaries $I A$ and $I B$ are essentially dual, and only the first is proved. Both imply Corollary $\mathrm{IC}$, which is due to Borges [2] and independently to Thomas [7]. Corollary iD appears to be a folk theorem. Both Corollaries IB and ID improve a result of Levine [6].

COROLLARY IA. Let $(X, \tau)$ be connected, and let $A \subseteq X$. If $X-A$ is connected and no component of $A$ is $\tau$-closed, then $(X, \tau(A))$ is connected.

Proof. If not, there exists nontrivial $C \subseteq X$ satisfying the conditions of Theorem 1 . By $(c)$ and $(d), C \cap(X-A)$ and $(X-C) \cap(X-A)$ are open relative to $X-A$. Thus either $X-A \subseteq X-C$ or $X-A \subseteq C$. If $X-A \subseteq X-C$, we have by (a) and (b) that $C \cap A=C$ is in $\tau \mid A$ and $(X-C) \cap A$ is in $\tau \mid A$, so that $C$ contains a component of $A$. But by 
(b) and (d): i-closed; hence this component of $A$ is $\tau$-closed, contrary to hyput. sis. Thus $X-A \subseteq C$. Again by $(a)$ and (b), $X-C$ contains a componeili of $A$. But by $(a)$ and $(c), X-C$ is $\tau$-closed, again a contradiction. Hence $(X, T(A))$ is connected.

COROLLARY 1B. Let $(X, \tau)$ be connected, and let $A \subseteq X$ be connected. If no union of components of $X-A$ is open in $X$, then $(X, \tau(A))$ is connected.

COROLLARY IC. Let $(X, \tau)$ be connected, and let $A \subseteq X$ be connected with connected complement. If $A$ is not closed, then $(X, \tau(A))$ is connected.

COROLLARY 10. Let $(X, \tau)$ be connected and let $A$ be dense in some $\tau$-open set. Then $(X, \tau(A))$ is connected.

Proof. Since $B=A \cup(X-\mathrm{Cl}(A))$ is dense in $X$ and $\tau(A)=\tau(B)$, it suffices to prove this in the case $A$ is dense in $X$. Suppose there exists nontrivial $C \subseteq X$ satisfying the conditions of the theorem. If $A \cap C=\emptyset$, then $C \subseteq X-A$ and by $(c), C$ is in $\tau$, while if $A \cap(X-C)=\varnothing$ then $A \subseteq C$ and by $(d), X-C$ is in $\tau$. Since $A$ is dense, $A \cap C \neq \varnothing \neq A \cap(X-C)$. Again using the density of $A,(a)$ and (c) together imply $\mathrm{Cl}(A \cap C)=C$ and (b) and (d) together imply $C l[A \cap(X-C)]=X-C$. Since this would be a disconnection of $(X, \tau)$, such $C$ cannot exist. Hence $(X, \tau(A))$ is connected.

Anderson [1] has obtained the following result for infinite expansions, which generalizes Corollary 10.

THEOREM 2. Let $(X, \tau)$ be connected, and let $A$ be a family of subsets of $X$ such that finite intersections of members of $A$ are dense in $X$. Then $(X, \tau(A))$ is connected.

\section{Maximally connected spaces}

Thomas [7] gave an example of a maximally connected $T_{1}$ space. It is an open question whether or not there exist maximally connected Hausdorff spaces other than singletons. Some aspects of this problem are studied below.

Bourbakl [3; p. 139] has defined the notion of a submaximal space and 
has characterized such spaces as those in which every dense set is open. Thus from Corollary 10 we deduce the following result.

THEOREM 3. Every maximally connected space is submaximal.

Bourbakl [3; p. 138] has also shown in effect that every topology can be expanded to a submaximal topology by adjoining an ultrafilter of dense sets. Together with Theorem 2 this yields another important result.

THEOREM 4. Every connected topology can be expanded to a connected submaximal topology.

EXAMPLE. Let $X=\{a, b, x, y\}$ with topology $\tau=\{\emptyset,\{a\},\{b\},\{a, b\},\{a, b, x\},\{a, b, y\}, x\}$. It is easy to verify that $(X, \tau)$ is a connected submaximal space which is not maximally connected.

A submaximal connected $T_{1}$ space is in particular a submaximal space without isolated points. The dispersion character [4] of a space is the least cardinal of a nonempty open set.

THEOREM 5. Let $X$ be a submaximal space with dispersion character $\Delta>1$. Then

(i) every discrete subspace is closed,

(ii) every subset of cardinal less than $\Delta$ is discrete,

(iii) no point has a local base of cardinal less than $\Delta$.

Proof. A discrete subspace $S$ with interior would produce an isolated point of $X$; hence $X-S$ is dense and therefore open. If $S$ has cardinal less than $\Delta$, every subset of $S$ has dense complement, so that $S$ is discrete. Finally, a choice function on deleted basic neighbourhoods of $p$ is a net converging to $p$. Since the range of this net is not closed, the base at $p$ must have at least $\Delta$ members.

Submaximal spaces without isolated points were studied in Kirch [5], where it is shown that their compact sets are finite. We employ similar methods to obtain slightly more precise results. A space is called $(m, n)$-compact ( $m, n$ infinite cardinals) if every open cover of cardinal at most $m$ admits a subcover of cardinal less than $n$.

THEOREM 6. Let $X$ be a submaximal space wi thout isolated points. 
Then every $(m, n)$-compact subset of $X$ has cardinal less than $n$.

Proof. If $K$ is an $(m, n)$-compact subset of $X$, then $K-\operatorname{int}(K)$ has empty interior, and so is closed and discrete. Then $K$ - int $(K)$ is $(m, n)$-compact, and since it is discrete it must have cardinal less than $n$. int $(K)$ can have no isolated points since $X$ has none; hence the set $I$ of isolated points of $K$ is contained in $K$ - int $(K)$ and so has cardinal less than $n$. Then $K-I$ is submaximal, $(m, n)$-compact, and free of isolated points. Let $k$ be the cardinal of $K-I$, and settheoretically partition $K-I$ into $k$ subsets of cardinal $k$. Then either one of the sets of the partition has empty interior, or a discrete set of cardinal $k$ can be constructed by choosing one point from the interior of each partitioning set. In any event, $K-I$ contains a closed, discrete, $(m, n)$-compact subset of cardinal $k$; hence $k<n$. It follows that $K$ has cardinal less than $n$.

COROLLARY 6A. If $X$ is a submaximal $T_{1}$ space without isolated points, then no point has a countable local base.

COROLLARY 6B. No topology is maximal anong connected first countable $T_{1}$ topologies.

\section{Subspaces}

We now show that maximal connectedness is hereditary to connected subspaces. This extends a result of Thomas [1] which we state as Lemma 1 below.

LEMMA 1. Every open connected subspace of a maximally connected space is maximally connected.

LEMMA 2. Every closed connected subspace of a maximally connected space is maximally connected.

Proof. Let $(X, \tau)$ be a maximally connected space and let $S$ be a closed connected subset of $X$. Suppose $(S, \tau \mid S)$ is not maximally connected. Then $S$ has some subset $B$ not in $\tau \mid S$ such that $(\tau \mid S)(B)$ is a connected topology for $S$. Let $A=X-(S-B)$, so that $A \cap S=B$. Then $(\tau \mid S)(B)=\tau(A) S$ and $\tau(A)$ is a proper expansion of $\tau$. Hence $X$ is disconnected by a partition $\{F, G\}$ of members of $\tau(A)$. Since 
$\tau(A) \mid S$ is connected topology, $S$ cannot meet both $F$ and $G$; let $S$ be a subset of $F$.

Let $x \in G$. Since $G$ is in $\tau(A)$, there are $V$ and $W$ in $\tau$ such that $x \in V \cup(W \cap A) \subseteq G$. If $x \in V \subseteq G$, it is a $\tau$-interior point of $G$. If $x \in W \cap A$, then since $x \in G \subseteq X-S \subseteq A$ we have $x \in W \cap(X-S) \subseteq W \cap A \subseteq G$. But $X-S$ is $\tau$-open, so again $x$ is a $\tau$-interior point of $G$. Thus $G$ is $T$-open.

Let $x \in F$. Then there are $V$ and $W$ in $\tau$ such that $x \in V \cup(W \cap A) \subseteq F$. If $x \in V \subseteq F$, then $x$ is a t-interior point of $F$. If $x \in W \cap A \subseteq F$, then since $W \cap(X-A) \subseteq X-A=S-B \subseteq S \subseteq F$ we have $W=W \cap[(X-A) \cup A] \subseteq F$. Then $x \in W \subseteq F$, and again $x$ is a t-interior point of $F$. Thus $F$ is $\tau$-open.

Now $F$ and $G$ are a $\tau$-open partition of $X$, a contradiction. Hence $(S, \tau \mid S)$ is maximally connected.

THEOREM 7. Every connected subspace of a maximally connected space is maximally connected.

Proof. Let $S$ be a connected subspace of a maximally connected space $X$. Then $\mathrm{Cl}(S)$ is connected in $X$, and by Lemma 2 is maximally connected. But $S$ is dense in $\mathrm{Cl}(S)$, so by Theorem $3, S$ is open in $\mathrm{Cl}(S)$. But then by Lemma $1, S$ is maximally connected.

COROLLARY 7A. Let $(X, \tau)$ be a maximally connected space, let $\rho$ be an expansion of $\tau$, and let $S$ be a p-component of $X$. Then $\rho|S=\tau| S$ and $S$ is maximally connected.

Proof. Clearly $\rho \mid S$ contains $\tau \mid S$. Since $\rho \mid S$ is a connected topology of $S$, so is $\tau \mid S$. Then by Theorem $7, \tau \mid S$ is a maximally connected topology for $S$, and hence $\tau|S=\rho| S$.

\section{References}

[1] Douglas R. Anderson, "On connected irresolvable Hausdorff spaces", Proc. Amer. Math. Soc. 16 (1965), 463-466.

[2] Carlos J.R. Borges, "On extensions of topologies", Canad. J. Math. 19 (1967), 474-487. 
[3] Nicolas Bourbaki, Elements of mathematics, General topology, Part 1 (Hermann, Paris; Addison-Wesley, Reading, Massachussetts; Palo Alto; London; Don Mills, Ontario; 1966).

[4] Edwin Hewitt, "A problem of set-theoretic topology", Duke Math. J. 10 (1943), 309-333.

[5] Murray R. Kirch, "A class of spaces in which compact sets are finite", Amer. Math. Monthly 76 (1969), 42.

[6] Norman Levine, "Simple extensions of topologies", Amer. Math. Monthly 71 (1964), 22-25.

[7] J. Pelham Thomas, "Maximal connected topologies", J. Austral. Math. Soc. 8 (1968), 700-705.

\footnotetext{
Department of Mathematics,

Universlty of Pittsburgh,

Pittsburgh,

Pennsylvania, USA;

Department of Mathematics,

West Virginia University,

Morgantown,

West Virginia, USA;

Department of Mathematics,

University of Pittsburgh,

Pittsburgh,

Pennsylvania, USA.
} 